\section{Tecido Adiposo como Glândula Endócrina}

\section{RESUMO}

O conceito de que os adipócitos são células secretórias surgiu nos últimos anos. Os adipócitos sintetizam e liberam uma variedade de peptídeos e não-peptídeos, bem com expressam outros fatores além de sua capacidade de depositar e mobilizar triglicerídios, retinóides e colesterol. Estas propriedades permitem uma interação do tecido adiposo como outros órgãos, bem como outras células adiposas. A observação importante de que adipócitos secretam leptina como o produto do gene ob estabeleceu o tecido adiposo como um órgão endócrino que se comunica com o sistema nervoso central. (Arq Bras Endocrinol Metab 2000;44/1: 13-20)

Unitermos: Tecido gorduroso; Hormônios secretantes; Fatores autócrinos e parácrinos

\begin{abstract}
The author has studied the hormonal secretion by the adipose tissue, related to fat metabolism, blood coagulation, steroids and energetic balance, such as leptin and adiponectin, and autocrine-paracrine relationships. (Arq Bras Endocrinol Metab 2000;44/1: 13-20)
\end{abstract}

Keywords: Fat tissue; Hormonal secretion; Autocrine-paracrine relationships

O CONCEITO DE QUE OS ADIPÓCITOS SÃo CÉLULAS secretórias surgiu nos últimos anos. Os adipócitos sintetizam e liberam uma variedade de peptídeos e não-peptídeos, bem como expressam outros fatores além de sua capacidade de depositar e mobilizar triglicerídeos, retinóides e colesterol. Estas propriedades permitem uma interação do tecido adiposo com outros órgãos, bem como com outras células adiposas. A observação importante de que os adipócitos secretam leptina como o produto do gene $a b$ estabeleceu o tecido adiposo como um órgão endócrino que se comunica com o sistema nervoso central.

Nas tabelas I e 2 estão indicados os fatores mais importantes liberados no tecido adiposo com ação endócrina/parácrina.

\section{A. PROTEÍNAS SECRETADAS E METABOLISMO DOS TRIGLICERIDEOS}

\section{Lipase lipoprotéica (LPL)}

A LPL é o regulador mais importante para a deposição dos triglicerídeos. A LPL hidrolisa os triglicerídeos das lipoproteínas de densidade muito baixa (VLDL) e quilomicra, liberando os ácidos graxos que são captados pelo adipócito. Os genes codificando a LDL não são expressos diferencialmente nos adipócitos omentais quando comparados aos subcutâneos (1),

\section{artigo original}

\author{
Bernardo Léo Wajchenberg
}


Tabela 1 - Fatores liberados no tecido adiposo

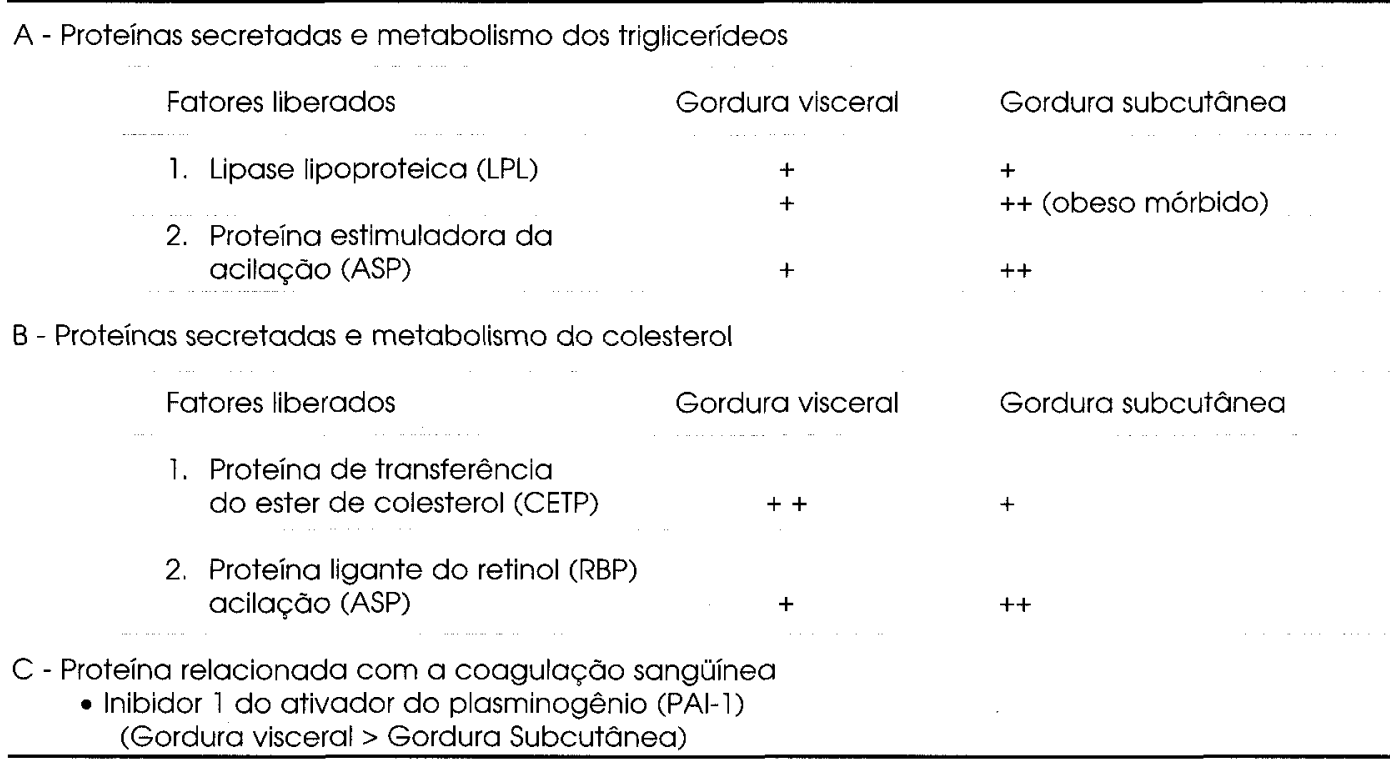

Tabela 2 - Fatores liberados no tecido adiposo

D - Fatores secretados com função endócrina

Fatores liberados

1. Estrogênios

2. Leptina

3. Angiotensinogênio

4. Adiponectina
Gordura visceral

$+$

$+$

$++$
Gordura subcutânea

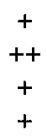

E - Fatores com atividade autócrina / parácrina de regulação da celularidade do tecido adiposo

Fatores liberados

1. Factor- $\alpha$ de necrose tumoral (TNF- $\alpha$ )

2. Interleucina - 6 (IL-6)

3. Fator de cresc.-1 insul-simile (IGF-1) Proteína ligante-3 do IGF (IGFBP3)

4. Monobutirina
Gordura visceral

+
+
+
+
+

Gordura subcutânea

+
+
+
+
+

mas nos obesos mórbidos o tecido gorduroso do omento (visceral) expressa o mRNA e a correspondente proteína em nível menor do que no subcutâneo (2). Quanto à regulação hormonal da LPL, a insulina e glicocorticóides são os estimuladores fisiológicos da atividade da LPL e a sua associação tem um papel importante na regulação da topografia da gordura corporal, sendo a LPL central para o desenvolvimento da obesidade visceral abdominal (3). Por outro lado, as catecolaminas, hormônio de crescimento e testosterona (no homem) reduzem a atividade da LPL do tecido adiposo (4).

\section{Estimuladora da acilação (ASP)}

A ASP é o estimulador mais potente da síntese de triglicerídeos no adipócito humano. $\mathrm{O}$ adipócito humano secreta 3 proteínas da via alternativa do complemento: $\mathrm{C} 3, \mathrm{~B}$ e fator $\mathrm{D}$ (adipsina) que interagem extracelularmente para produzir um peptídeo de 77 aminoácidos, a ASP (5). A medida em que os ácidos graxos são liberados das lipoproteínas ricas em triglicerídios e quilomicra, pela ação da LPL, a ASP é também produzida, a síntese de triglicerídeos aumentando conforme as necessidades. A via da ASP evitaria o excesso de formação dos ácidos graxos no lume capi- 
Tabela 3 - Fatores expressos no tecido adiposo

\begin{tabular}{lcc}
\hline Fatores liberados & Gordura visceral & Gordura subcutânea \\
$\begin{array}{l}\text { 1. Ativador do receptor } \gamma \text { do proliferador } \\
\text { peroxisomal (PPAR- } \gamma \text { ) }\end{array}$ & + & $+\ldots$ \\
& ++ & + (obeso) \\
2. Proteínas desacopladoras (UCPs) & & ++ \\
UCP - 1 & + & ++ \\
UCP - 2 & + & + \\
\hline
\end{tabular}

lar, os quilomicra sendo os determinantes da geração da ASP. A insulina aumenta a secreção da ASP, o que seria de se esperar pela ação concomitante da LPL e ASP. A expressão da ASP sendo maior no tecido subcutâneo do que no visceral, o tecido adiposo do omento teria uma capacidade limitada para impedir que os ácidos graxos cheguem ao fígado, o que poderia contribuir para as anormalidade metabólicas observadas na obesidade visceral $(6)$.

\section{B. PROTEÍNAS SECRETADAS E METABOLISMO DO COLESTEROL E RETINOL}

\section{Proteína de transferência do éster de colesterol (CETP)}

A CETP é um modulador importante do transporte reverso do colesterol por facilitar a transferência dos esteres de colesterol das lipoproteínas de elevada densidade (HDL) para as lipoproteínas aceptoras [contendo apoB, ricas em triglicerídios, particularmente as lipoproteínas de densidade muito baixa (VLDL)] e de triglicerídeos destas para as HDL. Em conseqüência, há um aumento da captação e degradação das HDL enriquecidas em triacilglicerol, por atividade da lipase protéica ao tempo que ocorre a captação pelo fígado das lipoproteínas contendo apoB enriquecidas em colesterol esterificado. Desta maneira, o tecido adiposo, sendo um órgão de depósito do colesterol, o colesterol periférico captado pelas HDL, que agem como aceptoras do efluxo do colesterol, retorna ao figado para ser excretado $(7,8)$. A síntese ou também a secreção da CETP no tecido adiposo está aumentada no jejum, dietas ricas em colesterol/gorduras saturadas, após o estimulo pela insulina e na obesidade. A correlação positiva da CETP circulante com a insulinemia e glicemia basais sugere uma relação com a resistência à insulina (9). Finalmente, a expressão da CETP na gordura do omento é maior do que na do tecido subcutâneo(7).

\section{Ligante do retinol (RBP)}

A RBP é sintetizada e secretada pelo adipócito (10), sendo que a transcrição do gene correspondente induzida pelo ácido retinóico (11), considerando que o tecido adiposo está envolvido no depósito e metabolismo do retinol. O mRNA codificando a RBP é expresso em nível relativamente elevado nos adipócitos sem haver diferença entre as células gordurosas do omento e subcutâneas (1). A mobilização do retinol dos depósitos gordurosos não parece estar relacionada com um aumento da secreção da RBP, mas à hidrólise de éster de retinol por uma lipase AMPc-dependente hormônio sensível (12).

\section{PROTEÍNA RELACIONADA COM A COAGULAÇĀO: INIBIDOR-1 DO ATIVADOR DO PLASMINOGÊNIO (PAI-1)}

O PAI-1 é uma protease, sendo um regulador muito importante do sistema fibrinolítico, uma defesa natural contra a trombose. Se liga e rapidamente inibe os ativadores do plasminogênio que modulam a fibrinólise endógena. Embora as fontes mais importantes do PAI-1 sejam os hepatócitos e células endoteliais, os adipócitos também contribuem para os níveis circulantes desta proteína, que estão elevados na obesidade, apresentando uma correlação elevada com os parâmetros que definem a resistência à insulina, particularmente a insulinemia $\mathrm{e}$ trigliceridemia basais, além do índice de massa corpórea e o acúmulo de gordura visceral, que explica $28 \%$ da variância da atividade do PAI-1 (13). Entretanto, o aumento do PAI-l na obesidade visceral é independente dos níveis de insulina, triglicerídeos e glicose circulantes (14). Estudos prospectivos mostraram a associação entre o aumento dos níveis de PAI- 1 e a correspondente fibrinólise defeituosa com o risco de ateroesclerose e trombose, particularmente em relação a eventos coronarianos (15), assim ligando o acúmulo de gordura visceral à moléstia macrovascular (13). 
A síntese de PAI-l no tecido adiposo é estimulada pelos corticosteróides (16) e significativamente relacionada com a do fator de necrose tumoral alfa (TNF-alfa), enfatizando a possível contribuição local desta citocina na regulação da produção de PAI-1 pelo tecido adiposo (17).

\section{FATORES SECRETADOS COM FUNÇĀO ENDÓCRINA}

\section{Estrogênios}

A atividade da aromatase P450 no tecido adiposo é importante para a produção de estrogênios (18). Com efeito, a estrona que é o segundo estrogênio predominante na circulação na mulher pré-menopausal e o predominante após a menopausa, é em sua maior parte derivada do metabolismo do estradiol secretado pelo ovário e da aromatização da androstenediona no tecido adiposo, antes da menopausa, e quase exclusivamente da aromatização deste andrógeno secretado pela adrenal, após a menopausa. Na mulher, a conversão da androstenediona a estrona, aumenta em função do envelhecimento e da obesidade, pelo aumento na transcrição da aromatase P450 na gordura subcutânea, nos adipócitos e células estromais (pré-adipócitos) (19-21), estimulada pela insulina e cortisol, nos pré - e adipócitos na mulher e somente adipócitos no homem, assim contribuindo para as diferenças sexuais no padrão da distribuição do tecido adiposo.

No homem, a conversão periférica da testosterona ao estradiol e da androstenediona a estrona estão aumentado na obesidade, bem como os correspondentes níveis circulantes (22). Desde que a conversão da testosterona ao estradiol não explica o aumento da produção do estradiol no homem obeso (22), é provável que o estradiol seja secretado ou resultado da conversão periférica da estrona ou também do sulfato de dehidroepiandrosterona, o esteróide adrenal mais abundante. Os andrógenos e estrógenos ativos produzidos localmente nos tecidos periféricos, especialmente o tecido adiposo, podem apresentar uma ação parácrina, interagindo com os correspondentes receptores nas mesmas ou células próximas onde ocorreu a sua síntese antes da sua liberação para o ambiente extracelular como tais ou metabólitos inativos (18).

\section{Leptina}

A leptina é o produto do gene da obesidade $(o b)$ que é expresso nos adipócitos (23). Estudos em roedores sugeriram que a leptina age como um fator de sinalização do tecido adiposo para o sistema nervoso central, regulando a ingestão alimentar e o gasto energéti- co e, assim, fazendo a homeostase do peso corporal e mantendo constante a quantidade de gordura (24).

No homem, como nos roedores, se observa uma correlação fortemente positiva entre os níveis circulantes de leptina e a quantidade de gordura corpórea, indicando a secreção de leptina ser um reflexo de hipertrofia gordurosa $(24,25)$. A leptina apresenta um ritmo circadiano, com valores noturnos mais elevados. $\mathrm{O}$ adipócito ć a única fonte conhecida do produto do gene $(o b)$, leptina, os pré-adipócitos não apresentando esta capacidade. A secreção de leptina é 2 a 3 vezes maior no tecido subcutâneo do que na gordura do omento particularmente $\mathrm{cm}$ mulheres do que nos homens, pelo fato dos adipócitos subcutâneos serem maiores do que os do omento, especialmente no sexo feminino, e daí maior expressão do gene da leptina (26).

A leptina é também regulada por outros fatores que não a massa do tecido adiposo, nutricionais e hormonais. Assim, alterações agudas no balanço energético regulam a expressão da leptina e os correspondentes nívcis circulantes. Assim, aumento na ingestão de hidratos de carbono induz uma elevação da leptina (secundária a hiperinsulinemia) em aproximadamente $40 \%$ nas primeiras 12 horas na ausência de alterações no peso corporal (27). Por outro lado, a ingestão isocalórica de gordura induz uma redução da leptina. $\mathrm{O}$ jejum prolongado induz uma queda na leptinemia em desproporção com as variações da massa de tecido adiposo (28). Portanto, em condiçǒes de equilíbrio energético, a leptina é um índice estático da quantidade de triglicerídeos no tecido adiposo e em situações de balanço energético em equilíbrio não estável a leptina, pode ser regulada agudamente de uma maneira independente dos depósitos de triglicerídeos e servindo com um sensor do balanço energético (24).

Quanto a regulação hormonal, a insulina e os glicocorticóides têm efeito estimulador nas concentrações circulantes de leptina (29), bem como os estrogênios e TNF-alfa (30). Por outro lado, as catecolaminas e androgênios reduzem os níveis de leptina. A leptina circulante exibe um dimorfismo sexual, com valores mais elevados na mulher, pela predominância do tecido gorduroso subcutâneo sobre o visceral e as condições hormonais prevalentes na mulher, estrogênios elevados e androgênios baixos (31).

O TNF-alfa modula positivamente a secreção de leptina pelos adipócitos, tendo-se demonstrado uma associação independente entre a leptinemia e o nível circulante do receptor solúvel $55 \mathrm{kDa}$ do TNF-alfa, um indicador sensível de ativação desta interleucina (32).

O sistema nervoso central é o sítio principal de ação da leptina, atuando em áreas específicas do 
hipotálamo e tronco cercbral, importantes na regulação da adiposidade corporal, como demonstrado em roedores. Assim, se observou a redução da expressão e liberação de peptídios orexigênicos, como o neuropeptídeo Y (33), melanocortina e galanina (34), com ativação do sistema nervoso simpático e aumento do gasto energético. Ao mesmo tempo, há um aumento dos peptídeos anorexigênicos hipotalâmicos: hormônio de liberação da corticotrofina $(\mathrm{CRH})$, peptídio glucagon-simile-1 (GLP-l) proópiomelanocortina (POMC) e o transcrito regulado pela cocaína e anfetamina (CART), levando a uma redução da ingesta alimentar e consequentemente da massa gordurosa (34). Além disso, a leptina afeta outros mecanismos neuroendócrinos que não a regulação da ingesta alimentar, como a atividade hipotálamo-pituitária, tal como no desencadeamento da puberdade em meninos (35).

A leptina também age nos tecidos periféricos. Assim, demonstrou-se em roedores, que a hiperleptinemia reduz a síntese de triglicerídeos e aumenta a oxidação dos ácidos graxos em ilhotas pancreáticas normais (36), levando a uma disfunção das células beta, deprivando-as do sinal lipídico necessário para a resposta insulínica aos secretagogos (37). Este achado, em conjunto com a observação de que a insulina estimula a secreção de leptina, sugeriu a possível existência de uma regulação retrógrada negativa entre a insulina e a leptina.

\section{Angiotensinogênio}

$\mathrm{O}$ angiotensinogênio, sintetizado primariamente no fígado, é também secretado em abundância pelo tecido adiposo, onde a expressão gênica é regulada pelos glicocorticóides (38). A expressão do angiotensinogênio é similar na massa visceral e subcutânea. $O$ angiotensinogênio é clivado pela renina à angiotensina I e esta convertida a angiotensina II pela enzima de conversão da angiotensina, ambas as enzimas também expressas no tecido adiposo (39). Assim, a angiotensina II, produzida localmente no tecido adiposo, estimulando a produção de prostaciclina pelos adipócitos pode induzir a diferenciação dos pré-adipocitos a adipocitos (4). Além do efeito no desenvolvimento do tecido adiposo, o aumento da secreção do angiotensinogênio, via angiotensina II, poderia induzir um aumento da tensão arterial observado com freqüência na obesidade (4).

\section{Adiponectina}

A adiponectina, uma proteína secretante colágenosímile, é especifica e abundantemente expressa no tecido adiposo, com predominância na gordura visceral, sendo detectada no plasma humano, correlacionandose negativamente com o índice de massa corporal e área da gordura visceral abdominal, porém não com a gordura subcutânea abdominal (40). Estudos em macacos rhesus mostraram que havia uma correlação inversa significativa entre o peso corporal e os valores de adiponectina plasmática ao contrário do verificado para a leptina circulante. Em macacos hiperinsulinêmicos, os níveis de adiponectina estavam reduzidos enquanto os de leptina, elevados. Estudos longitudinais mostraram que a adiponectina circulante era negativamente regulada pela adiposidade (41). Finalmente, em pacientes com moléstia coronariana se observaram valores mais baixos do que nos controles, independente do índice de massa corporal ou gordura visceral (42).

\section{E. Fatores com atividade autócrina/parácrina regulando a celularidade do tecido adiposo}

\section{Fator de necrose tumoral-alfa (TNF-alfa)}

O TNF-alfa é expresso nos adipócitos e estando ausente nos pré-adipócitos, havendo uma correlação significativa entre o mRNA TNF-alfa, e a correspondente proteína circulante com o nível de insulina (medida indireta da resistência à insulina) e o índice de massa corpórea $(43,44)$. Os genes codificando esta citocina não são expressos diferencialmente no tecido adiposo subcutâneo e omental (1). O TNF-alfa se correlaciona negativamente com a atividade da lipase lipoprotéica no tecido gorduroso, havendo indicações de que esta citocina teria um efeito local, regulando o tamanho do adipócito em face do aumento de consumo energético, ou seja, representando uma forma de "adipostato" $(43,45)$. Assim, além de diminuir a atividade da LPL, o TNF-alfa reduz a expressão do transportador de glicose GLUT 4 $\mathrm{e}$ induz o aumento da lipase hormônio-sensivel (46).

Em pacientes do sexo masculino, com moléstia coronariana prematura, mostrou-se haver aumento do TNF-alfa circulante, sem relação com os indicadores de resistência à insulina mas associados com distúrbios metabólicos aterogênicos (47). Com efeito, observações no tecido adiposo subcutâneo de homens magros e obesos diabéticos, do tipo 2 ou não, mostraram que a expressão do mRNA do TNF-alfa foi normal nos diversos grupos de pacientes em comparação aos controles sem relação com a hiperinsulinemia e não estando associada com a obesidade ou resistência à insulina (48).

\section{Interleucina-6 (IL-6)}

A IL-6, produzida pelo tecido adiposo, tem a sua concentração plasmática proporcional à massa de gordura (49). O TNF-alfa induz a produçăo de IL-6 pelos 
adipócitos, maior pela gordura omental do que pela subcutânea (50). Entre as ações da IL-6 está a redução da atividade da lipase lipoprotéica, assim mostrando uma ação local na regulação da captação dos ácidos graxos pelo tecido adiposo, em conjunto com o TNFalfa ("adipostato"), resultando em maior afluxo de ácidos graxos para o fígado, no caso da gordura visceral abdominal, que é de particular importância, considerando que a IL-6 aumenta a secreção de triglicerídios pelo figado (51). Assim, esta citocina contribuiria para a hipertrigliceridemia associada com a obesidade visceral. A IL-6, além de ser um regulador autócrino e parácrino da função do adipócito, tem efeitos em outros tecidos, como estimulando a síntese de proteínas da fase aguda e estimulando o eixo hipotálamo-pituitária-adrenal (52) e, por outro lado, os glicocorticóides reduzem a produção de IL-6 (53), assim agindo como um regulador deste eixo de importância no metabolismo acelerado de cortisol, uma característica da obesidade visceral.

\section{Fator de crescimento insulino-simile-1 (IGF- 1) e proteína 3 ligante do IGF (IGFBP 3)}

Demonstrou-se que a diferenciação de pré-adipócitos está associada com um aumento no IGF-1 e na proteína 3 ligante do IGF (IGFBP-3), independente da presença do hormônio de crescimento (53). Em adipócitos diferenciados, o hormônio de crescimento humano estimula a expressão e produção da IGFBP-3, mas não do IGF-1. Existem, portanto, evidências da ação autócrina/parácrina do IGF-l e IGFBP-3 no tecido adiposo humano com modulação pelo hormônio de crescimento e cortisol (que reduz a expressão e produção da IGFBP-3 em adipócitos). Portanto, em pré-adipócitos, a produção de IGF-1 atuando de maneira autócrina/parácrina induz a proliferação dos pré-adipócitos e sua diferenciação em adipócitos $(54,4)$.

\section{Monobutirina}

A monobutirina (1-butiril-glicerol) é um produto de secreção do adipócito, favorecendo a vascularização do tecido adiposo no seu desenvolvimento e vasodilatação dos microvasos (55).

\section{REFERÊNCIAS}

1. Montague CT, Prins JB, Sanders L, Zhang J, Sewter CP, Digby $J$, et al. Deprot-related gene expression in human subcutaneous and omental adipocytes. Diabetes 1998:47:1384-91.

2. Fried SK, Russell CD, Grauso NL, Brolin RE. Lipoprotein lipase regulation by insulin and glucocorticoid in subcu- taneous and omental adipose tissues of obese women and men. J Clin Invest 1993:92:2191-8.

3. Kern PA. High adipose tissue lipoprotein lipase activity plays a causal role in the etiology of obesity. In: Angel $A$, Anderson $H$, Bouchard C, Lau D, Leiter L, Mendelson $R$ (eds) Progress in Obesity Research: 7 Proceed $7^{\text {th }}$ Internat Congress on Obesity (Toronto, Canada. 20-25 august 1994 ) John Libbey \& Company, London, 1996, pp. 89-94

4. Ailhaud $G$. L'adipocyte, cellule sécrétice et endocrine. Médecine /Sciences 1998; 14:858-64.

5. Sniderman AD, Julien $P$, Ciaflone $K$. Peripheral triglyceride clearance, the Adipsin-ASP pathway and Type IV hyperlipoproteinemia. In: Year Book of Endocrinol. Bagdade JD (ed.) , Mosby-year Book, Inc, St. Louis, Missouri, pp XIX-XXIII, 1995.

6. Maslowska MH, Sniderman AD, MacLean LD, Cianflone $\mathrm{K}$. Reglonal differences in triacyglycerol synthesis in adipose tissue. J Lip Res 1993:34:219-28.

7. Angel $A$, Shen $G$. Secretion of cholesteryl ester transfer protein by adipose tissue In: Angel $A$, Anderson $H$, Bouchard C, Lau D, Leiter L, Mendelson R (eds) Progress in Obesity Research: 7 Proceed $7^{\text {th }}$ Internat Congress on Obesity (Toronto, Canada 20-25 august 1994) John Libbey \& Company, London, 1996, pp. 13-9.

8. Tall AR. Plasma cholesteryl ester transfer protein. J Lipid Res 1993:34: 1255-74.

9. Dullaart RP, Sluiter WJ, Dikkeschei LD, Hoogenberg K, Van Tol A. Effect of adiposity on plasma lipid transfer protein activities: a possible link between insulin resistance and high density lipoprotein metabolism. Eur $\mathbf{J}$ Clin Invest 1994:24:188-94.

10. Tsutsumi $C$, Okuno M, Tannous L, Piantedosi R, Allan M, Goodman DS, et al. Retinoids and retinoid-binding protein expression in rat adipocytes. J Biol Chem 1992:267:1805-10.

11. Okno M, Caraveo VE, Goodman DS, Blanner WS. Regulation of adipocyte gene expression by retinoic acid and hormones: effects on the gene encoding cellular retinol-binding protein J Lipid Res 1995;36:137-47.

12. Wei S, Lai K, Patel S, Piantedosi R, Shen $H$, Colantuoni V, et al. Retinyl ester hydrolisys and retinol efflux from BFC1 beta adipocytes. J Biol Chem 1997;272: 14159-65.

13. Alessi MC, Peiretti F, Morange P. Henry M, Nalbone $G$, Juhan-Vague I. Production of Plasminogen activator inhibitor 1 by human adipose tissue. Possible link between visceral fat accumulation and vascular disease. Diabetes 1997;46:860-7.

14. Janand-Delenne B, Chagnaud C, Raccah D, Alessi MC, Juhan-Vague I, Vague $P$. Visceral fat as a main determinant of Plasminogen activator inhibitor-? level in women. Int J Obes 1998:22:312-7.

15. Panahloo A, Yudkin JS. Diminished fibrinolysis in diabetes mellitus and its implication for diabetic vascular disease. Coronary Artery Disease 1996;7:723-31

16. Alessi MC, Morange P, Ventura N, Aubert J, Négrel R, Juan-Vague |. Corticosteroids and insulin affect PAl-1 synthesis by human adipose tissue. Eighth Internacional Congress on obesity (Paris, France, 29 august- 3 september 1998), Int J Obes 1998;22(Suppl 3):0255, p \$71.

17. Morange $P$, Alessi MC, Ventura N, Verdier M, Casanova 
D. Magalon $G$, et al. PAl- 1 antigen production by human adipose tissue is correlated with that of TNFalpha Eighth International Congress on Obesity (ParisFrance,29 august - 3 september 1998). Int J Obes 1998:22(Suppl 3):P31, ps 103.

18. Mizutani T, Nishikawa Y, Adachi $\mathrm{H}$, Enomoto T, lkegami $\mathrm{H}$, Kurachi $\mathrm{H}$, et al. Identification of estrogen receptor in human adipose tissue and adipocytes. J Clin Endocrinol Metab 1994;78:950-4.

19. Cleland WH, Mendelson CR, Simpson ER. Effects of aging and obesity on aromatase activity of human adipose cells. J Clin Endocrinol Metab 1985:38:476-9.

20. Bulun SE, Simpson ER. Competitive reverse transcriptionpolymerase chain reaction analysis indicates that levels of aromatase cytochrome P450 transcripts in adipose tissue of buttocks, thighs and abdomen of women increase with advancing age. J Clin Endocrinol Metab $1994 ; 78: 428-32$

21. Siteri PK. Adipose tissue as a source of hormones. Am J Clin Nutr 1987:47:277-82.

22. Schneider $G$, Kirschner MA, Berkowitz $R$, Hertel NH, Increased estrogen production in obese men. J Clin Endocrinol Metab 1979:48:633-8.

23. Halaas JL, Gajiwala KS, Maffel M, Cohen SL, Chait BT, Rabinowitz $D$, et al. Weight-reducing effects of the plasma protein encoded by the obese gene. Science 1995;269:543-6

24. Caro JF, Sinha MK, Kolaczynski JW, Zhang PL, Considine RV. Leptin: the tale of an obesity gene. Diabetes 1996:45: 1455-62.

25. Maffei $M$, Halaas J, Ravussin E, Pratley RE, Lee GH, Zhang $Y$, et al. Leptin levels in human and rodent: measurement of plasmo leptin and obRNA in obese and weightreduced subjects. Nat Med 1995;1:1155-61.

26. Van Harmelen $V$, Reynisdottir $S$, Eriksson $P$, Thörne $A$ Hoffstedt J, Lönnqvist F, et al. Leptin secretion from subcutaneous and visceral adipose tissue in women. Diabetes 1998:47:913-7.

27. Kolaczynski JW, Ohannesian J, Considine RV. Response to leptin to short-term and prolonged overfeeding in humans. J Clin Endocrinol Metab 1996:81:4162-5.

28. Boden $G$, Chen X, Mozzoli M. Effect of fasting on serum leptin in normal human subjects. J Clin Endocrinol Metab 1996:81:3419-23.

29. Halleux CM, Servais I, Reul BA, Detry R, Brichard SM. Multihormonal control of ob gene expression and leptin secretion from cultured human visceral adipose tissue increased responsiveness to glucocorticolds in obesity. J Clin Endocrinol Metab 1998:83:902-10.

30. Kirchgessner TG, Uysal T, Wiesbrock SM, Marino MW Hotomisligil $G$. Tumor necrosis factor-alpha contributes to obesity-related hyperleptinemia by regulating leptin release from adipocytes. J Clin Invest 1997; 100:2777-82.

31. Elbers $H M H$, Asscheman $H$, Seidell JC, Frölich $M$, Meinders $A E$, Gooren LJG. Reversal of the sex difference in serum leptin levels upon cross-sex hormone administration in transsexuals. J Clin Endocrinol Metab $1997 ; 82: 3267-70$.

32. Montzoros CS, Moschos S, Avramopoulos I, Kaklamani V Liolios A Doulgerakis DE, et al. Leptin concentrations in relation to body mass index and the tumor necrosis factor-alpha system in humans. J Clin Endocrinol Metab 1997:82:3408-13.

33. Tritos NA, Mantzoros CS. Leptin: its role in obesity and beyond. Diabetologia 1997:40:1371-9.

34. Kalra SP, Dube MG, PU OS, Xu B, Horvath TL, Kalra PS Interacting appetite-regulating pathways in the hypothalamic regulation of body weight. Endocrine Rev 1999:20:68-100.

35. Mantzoros CS, Flier JS, Rogol AD. A longitudinal assessment of hormonal and physical alterations during normal puberty in boys vs. rising leptin levels may signal the onset of puberty. J Clin Endocrinol Metab 1997:82:106670 .

36. Shimabukuro M, Koyama K, Chen G, Wang M-Y, Trieu F Lee $Y$, et al. Direct antidiabetic effect of leptin through triglyceride depletion of tissues. Proc Natl Acad Sci USA 1997:94:4637-41.

37. Koyama K, Chen G, Wang M-Y, Lee $Y$, Shimabukuro $M$, Newgard $C B$, et al. Beta-cell function in normal rats made chronically hyperleptinemic by adenovirus-leptin gene therapy. Diabetes 1997:46:1276-80.

38. Aubert J, Darimont $C$. Safonova I, Allhaud $G$, Négrel $R$. Regulation by glucocorticoids of angiotensinogen gene expression and secretion in adipose cells. Biochem J 1997,328:701-6.

39. Karlsson C, Lindell K, Ottoson M. Sjöström L, Carlsson B, Carlsson L MS. Human adipose tissue expresses angiotensinogen and enzymes required for its conversion to angiotensin II. J Clin Endocrinol Metab 1998:83:3925-9.

40. Kihara S, Arita $Y$, Ouchi N, Maeda K, Masahiko T, Yamashita $S$, et al. A novel adipocyte-derived factor, adiponectin, inhibits growth of vascular smooth muscle cell. Eighth International Congress on Obesity (Paris, France, 29 august - 3 september 1998). Int J Obes 1998:22(Supp| 3) O16, pS5.

41. Hotta K, Bodkin NL, Arita Y, Muraguchi M, Ortmeyer HK, Funahashi T, et al. Analysis of plasma adiponectin and leptin levels in rhesus monkeys. Eighth International Congress on Obesity (Paris, France, 29 august - 3 september 1998). Int J Obes 1998:22(Suppl 3) P 249, pS 162.

42. Arita $Y$, Kihara $S$, Funahashi T, Takahashi $M$, Ouchi N, Yamashita $S$, et al. A novel adipocyte-derived factor, adiponectin, is decreased in obesity and coronary artery disease. Eighth International Congress on Obesity (Paris, France, 29 august - 3 september 1998). Int J Obes 1998:22 (Suppl 3) 018, pS S42.

43. Kern PA, Saghizadeh M, Ong JM, Bosch RJ, Deem R, Simsolo RB. The expression of tumor necrosis factor in human adipose tissue. Regulation by obesity, weight loss, and relationship to lipoprotein lipase. Clin Invest 1995:95:2171-29.

44. Hotamisligil GS, Arner P, Caro JF, Atkinson RL, Spiegelman BM. Increased adipose tissue expression of tumor necrosis factor-alpha in human obesity and insulin resistance J Clin Invest 1995:95:2409-15.

45. Spiegelman BM, Hotamisligil. Through thlck and thin: wasting, obesity, and TNF-alpha. Cell 1993;73:625-7.

46. Patton JS, Shepard HM, Wilking $H$, Lewis $G$, Aggarwal BB, Eessalu $T E$, et al. Interferons and tumor necrosis factors 
have similar catabolic effects on 3T3-L1 cells. Proc Nat Acad Sci USA 1986:83:8313-7.

47. Jovinge S, Hamsten A, Tornvall P, Proudler A, Bävenholm $P$, Ericsson $C-G$, et al. Evidence for a role of tumor necrosis factor-alpha in disturbances of triglyceride and glucose metabolism predisposing to coronary heart disease. Metabolism 1998:47:1 13-8.

48. Koistinen HA, Koivisto VA, Ebeling P, Wallier P, Perche S, Riou J-P, et al. The subcutaneous adipose tissue expression of the TNF-alpha is not related to obesity or insulin resistance in humans. Eighth International Congress on Obesity (Paris, France, 29 august - 3 september), Int J Obes 1998;22 (Suppl 3) O227, pS 561.

49. Vgontzas NA, Papanicolaou DA, Bixler EO, Kales A, Tyson K. Crousos GP. Elevation of plasma cytokines in disorders of excessive daytime sleepiness: role of sleep disturbance and obesity. J Clin Endocrinol Metab 1997:82:1313-6.

50. Fried SK, Bunkin DA, Greenberg AS. Omental and subcutaneous adipose tissues of obese subjects release interleukin-6: depot difference and regulation by glucocorticoid. J Clin Endocrinol Metab 1998;83:847-50.

51. Nonogaki K, Fuller GM, Fuentes NL, Moser AH, Staprans I. Grunfeld $C$. Interleukin- 6 stimulates hepatic triglyceride secretion in rats. Endocrinology 1995; 136:2143-9.
52. Päth $G$, Bornstein SR, Ehrhart-Bornstein $M$, Scherbaum WA. Interleukin-6 and the interleukin-6 receptor in the human adrenal gland: expression and effects on steroidogenesis. J Clin Endocrinol Metab 1997:82:2343-9.

53. Wabitsch $M$, Schmid S, Braun $M$, Christofferesen $C T$, Torqvist H, Blum WF, et al. Expression of Insulin-like growth factor-1 (IGF-1), IGF-1 receptor and IGF-binding protein 3 in human adipocyte precursor cells during differentiation. Eighth International Congress on Obesity (Paris, France, 29 august - 3 september 1998). Int J Obes 1998:22 (Suppl 3) P 11, p S98.

54. Kamay Y, Mikawa S, Endo K, Sakai H, Komano T. Regulation of insulin-like growth factor-l expression in mouse preadipocyte Ob1771. J Biol Chem 1996:271:9883-6.

55. Wilkinson WE, Spiegelman BM. Biosynthesis of the vasoactive lipid monobutyrin. J Biol Chem $1993 ; 268: 2844-59$.

\section{Endereço para correspondência:}

Bernardo Léo Wajchenberg

Al. Gabriel Monteiro Silva, $n^{\circ} 2607$

Bela Vista

0144 1-002 - São Paulo, SP

e-mail: cliendo@warp.com.br 